\title{
Atomic-Scale Study of Grain Boundary Structures in Poly-Crystalline CdTe Solar Cells using Abberation-Corrected STEM
}

\author{
Tadas Paulauskas ${ }^{1}$, Rober F. Klie ${ }^{1}$, Zhao Guo ${ }^{1}$, Eric Colegrove ${ }^{1,2}$ \\ 1. University of Illinois at Chicago, Department of Physics \\ 2. EPIR Technologies, Inc.
}

Sunlight is a compelling solution to our need for clean and abundant sources of energy in the future. In order for the solar derived energy to claim a larger share of the world's total produced energy it is necessary to reduce the cost per watt of delivered solar electricity. Poly-crystalline thin film CdTe photo-voltaic devices have a huge potential due to high sunlight absorption coefficient and ideal direct band gap on top of the lowest manufacturing costs in today's solar industry. However, CdTe device conversion efficiency has achieved very minor improvements over the last 20 years with the current state-of-the-art $18.3 \%$.[1] One of the main concerns is a detrimental effect of grain boundaries which can provide charge carrier recombination centers and impinge charge transport. Here we present studies of the defect structures in an attempt to create an atomistic model of dislocation cores which could then be further examined via first-principles calculations to gain a deeper understanding of the defect role.

In this study, we use CdTe thin films grown by molecular beam epitaxy (MBE) on patterned and bicrystal substrates with well-defined grain boundaries. Aberration-corrected cold-field emission scanning transmission electron microscopy (STEM) analysis will be carried out in the JEOL JEMARM200CF using atomic-scale high-angle annular dark field (HAADF) and annular bright field (ABF) imaging. Chemical composition and electronic environment of bulk and grain boundaries is examined via X-ray energy dispersive (XEDS) and electron energy-loss spectroscopies (EELS).

Figure 1a) shows a low magnification bright field STEM images of a poly-crystalline CdTe PV device grown by close-space sublimation (CSS). The image clearly shows the larger grains of CdTe on top of the $\mathrm{CdS}$ and transparent conductive oxides layers. The CdTe grain size is estimated to be around $2 \mu \mathrm{m}$, and each grain exhibits additional contrast, which has been attributed to twin boundaries and point defects. Despite the high concentration of defects, such devices still reach a conversion efficiency of $15.3 \%$. (Figure 1b).[2] Figure 2a) shows EELS spectra taken from the bulk of a CdTe grain and from a grain boundary. It appears that the fine-strucutre of both the $\mathrm{Cd} M$ - and $\mathrm{Te} M$-edges is very similar in both spectra, and we did not find any sign of increased inpurity concentration, such as $\mathrm{O}$ or $\mathrm{N}$ at the grain boundary. Figure 2b) shows an atomic-resolution HAADF image of a twin boundary in CdTe (110) and preliminary EELS analysis appears to indicate changes in the local stoichiometry across such twin boundaries.

In this presentation, we will demonstrate the effects of twin boundaries and point defects on the local atomic and electronic strucutres, and examine low angle tilt grain boundaries in CdTe thin films. In addition, we will explore the effects of $\mathrm{CdCl} 2$ treatment on the local grain morphology and try to quantify the concentration of $\mathrm{Cl}$ as a function of defect structure. [3] 
References:

[1] Best research cell efficiency, www.nrel.gov

[2] Colegrove Eric et al, High-Efficiency Polycrystalline CdS/CdTe Solar Cells on Buffered Commercial TCO-Coated Glass. Journal of Electronic Materials, 2012. 41(10): p. 2833-2837.

[3] This research is supported by a grant from the Department of Energy Sunshot Program (DOE DEEE0005956)
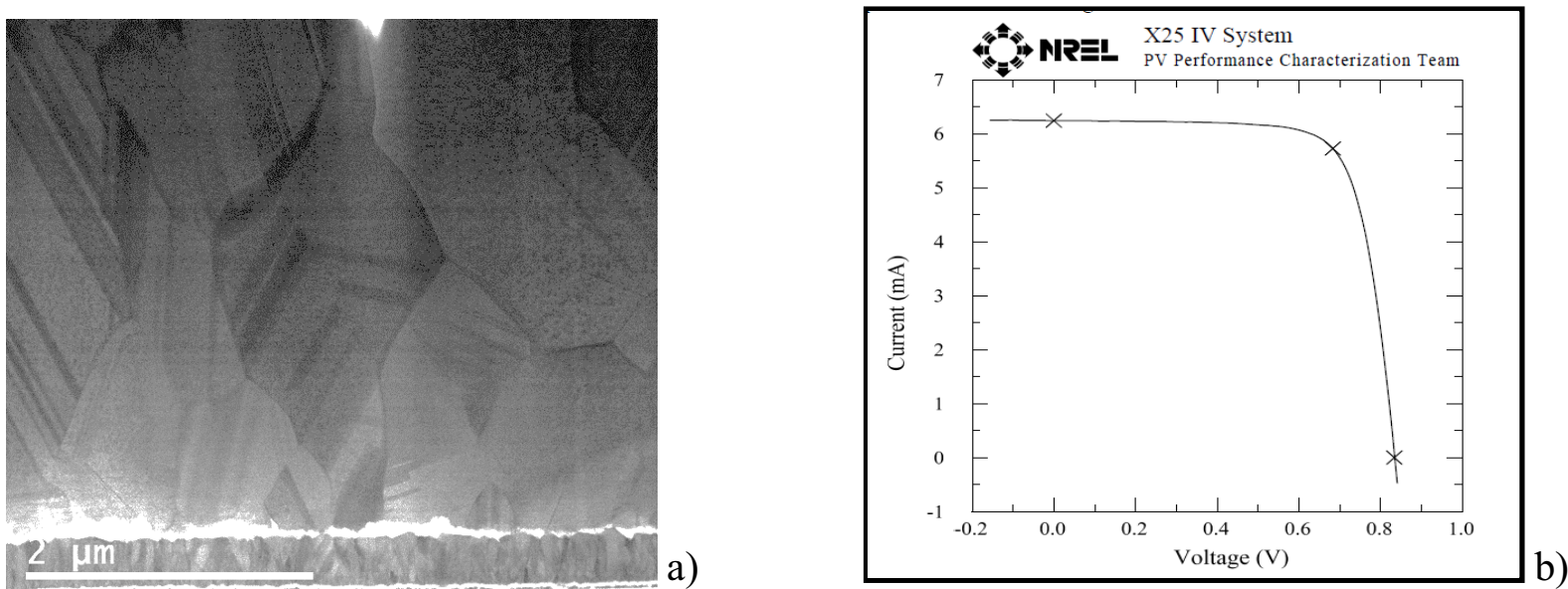

Figure 1. a) Low magnification HAADF image of a poly-crystalline CdTe solar cell. Multiple grains and twin boundaries can be seen in the CdTe layer. b) National Renewable Energy Laboratory verified I-V curve showing one of the highest achieved efficiencies.
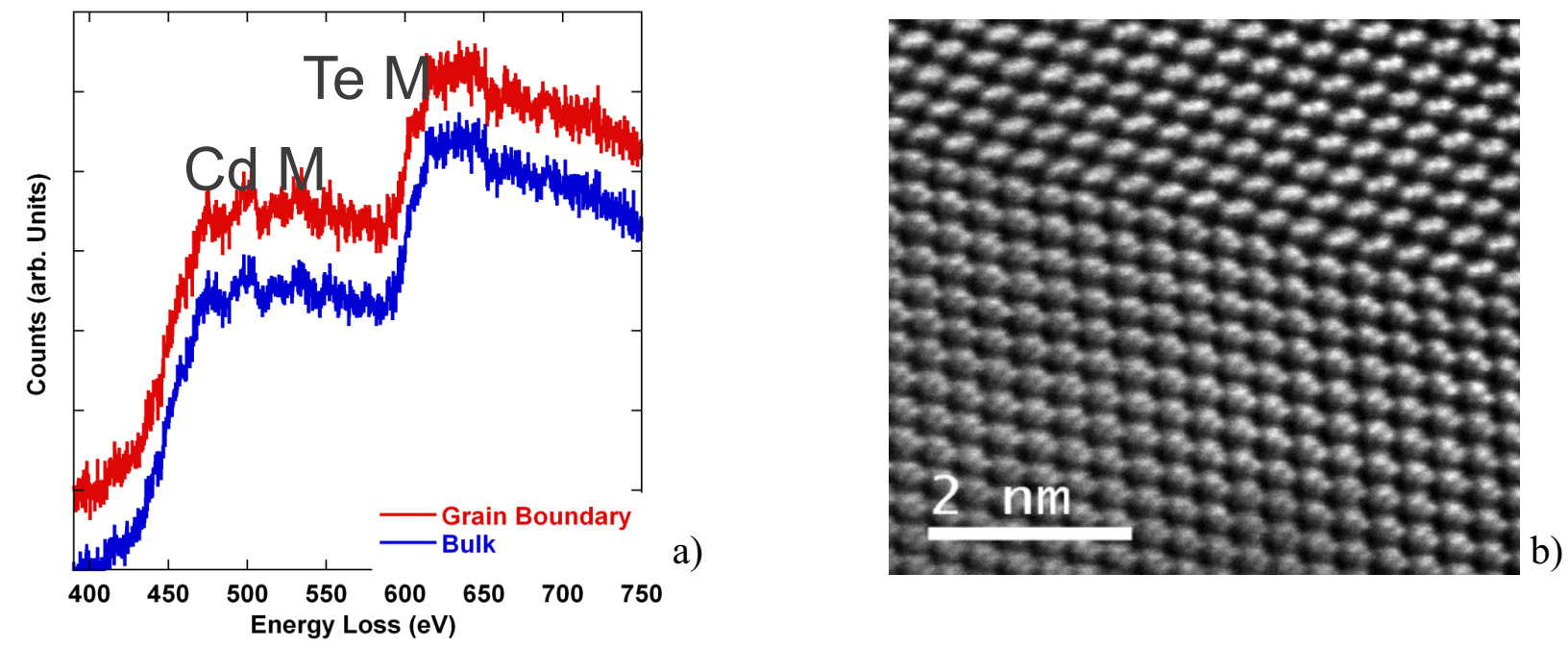

Figure 2. a) EELS spectra of Cd and Te M-edges as taken from bulk and a grain boundary. b) Atomic resolution Z-contrast image of a twin boundary along [110] zone axis. 